\title{
Extended Main-sequence Turnoff as a Common Feature of Milky Way Open Clusters
}

\author{
G. Cordoni $^{1}$ (D) A. P. Milone ${ }^{1}$ (D) , A. F. Marino ${ }^{1,2}$ (D) , M. Di Criscienzo ${ }^{3}$, F. D’ Antona ${ }^{3}$, A. Dotter ${ }^{4}$ (D) E. P. Lagioia ${ }^{1}$ (D) , and M. Tailo ${ }^{1}$ \\ ${ }^{1}$ Dipartimento di Fisica e Astronomia "Galileo Galilei”-Univ. di Padova, Vicolo dell'Osservatorio 3, Padova, I-35122, Italy; giacomo.cordoni@phd.unipd.it \\ ${ }^{2}$ Research School of Astronomy \& Astrophysics, Australian National University, Canberra, ACT 2611, Australia \\ ${ }^{3}$ Istituto Nazionale di Astrofisica-Osservatorio Astronomico di Roma, Via Frascati 33, I-00040 Monteporzio Catone, Roma, Italy \\ ${ }^{4}$ Harvard-Smithsonian Center for Astrophysics, 60 Garden Street, Cambridge, MA 02138, USA \\ Received 2018 October 1; revised 2018 October 30; accepted 2018 October 31; published 2018 December 19
}

\begin{abstract}
We present photometric analysis of 12 Galactic open clusters and show that the same multiple-population phenomenon observed in Magellanic Clouds (MCs) is present in nearby open clusters. Nearly all the clusters younger than $\sim 2.5 \mathrm{Gyr}$ of both MCs exhibit extended main-sequence turnoffs (eMSTOs) and all the cluster younger than $\sim 700 \mathrm{Myr}$ show broadened/split main sequences (MSs). High-resolution spectroscopy has revealed that these clusters host stars with a large spread in the observed projected rotations. In addition to rotation, internal age variation is indicated as possibly responsible for the eMSTOs, making these systems the possible young counterparts of globular clusters with multiple populations. Recent work has shown that the eMSTO + broadened MSs are not a peculiarity of MCs clusters. Similar photometric features have been discovered in a few Galactic open clusters, challenging the idea that the color-magnitude diagrams (CMDs) of these systems are similar to single isochrones and opening new windows to explore the eMSTO phenomenon. We exploit photometry+proper motions from Gaia DR2 to investigate the CMDs of open clusters younger than $~ 1.5$ Gyr. Our analysis suggests that: (i) 12 open clusters show eMSTOs and/or broadened MSs, which cannot be due to either field contamination or binaries; (ii) split/broadened MSs are observed in clusters younger than $\sim 700 \mathrm{Myr}$, while older objects display only an eMSTO, similarly to MCs clusters; (iii) the eMSTO, if interpreted as a pure age spread, increases with age, following the relation observed in MCs clusters and demonstrating that rotation is responsible for this phenomenon.
\end{abstract}

Key words: globular clusters: general - Hertzsprung-Russell and C-M diagrams - open clusters and associations: general - stars: abundances - stars: Population II - techniques: photometric

\section{Introduction}

In the past years, work based on high-precision Hubble Space Telescope (HST) photometry discovered that the colormagnitude diagrams (CMDs) of most star clusters younger than 2.5 Gyr in the Large and Small Magellanic Cloud (LMC and SMC) are not consistent with simple stellar populations. Specifically, most, if not all, of them exhibit extended mainsequence turnoffs (eMSTOs, e.g., Mackey \& Broby Nielsen 2007; Glatt et al. 2008; Milone et al. 2009), and clusters younger than $\sim 700$ Myr display both eMSTOs and split main sequences (MSs, e.g., Milone et al. 2013; 2015; 2018, Correnti et al. 2017; Li et al. 2017).

The comparison between the observed and synthetic CMDs from the Geneva database (e.g., Georgy et al. 2014) suggests that split MSs are consistent with two stellar populations with different rotation rates. A group of stars with rotation close to the breakout value $\left(\omega \sim 0.9 \omega_{\mathrm{cr}}\right)$, which corresponds to the red MS and includes about two-thirds of the total number of MS stars, and a population of slow rotators with $\omega \sim 0$, which populate the blue MS (e.g., D'Antona et al. 2015; Milone et al. $2016 \mathrm{~b}$ ). On the turn-off region, rapidly and slowly rotating stars distribute on brighter and fainter magnitudes, respectively. Measurements of rotational velocities in MS stars of the LMC cluster NGC 1818 from high-resolution spectra collected with the Very Large Telescope (VLT) has recently provided direct evidence that the red-MS and the blue-MS stars exhibit different rotation rates (Marino et al. 2018a). Similarly, highresolution Magellan spectra confirm that the bright and the faint MSTO of NGC 1866 are mostly populated by slow and fast rotators, respectively (Dupree et al. 2017).
Although it is now widely accepted that rotation is one of the main drivers for the photometric features appearing on the CMDs of young and intermediate-age MC clusters, it might not be able to entirely reproduce the observations. Indeed, as noticed by Milone et al. (2017), a fraction of eMSTO are consistent with being younger than the bulk of cluster stars. It has been suggested that some clusters have experienced a prolonged star formation, and that age variation, together with rotation is responsible for the eMSTOs (e.g., Goudfrooij et al. 2014, 2017). In this case, the MC clusters could represent the younger counterparts of the old globular clusters with multiple populations (e.g., Conroy \& Spergel 2011; Keller et al. 2011). As an alternative, D'Antona et al. (2017) suggested that the evolution of braked rapidly-rotating stars can mimic an age spread and contribute to the eMSTO.

The recent discovery of eMSTOs in four open clusters, namely NGC 2099, NGC 2360, NGC 2818, and NGC 6705 has challenged the text-book concept that the CMDs of open clusters are proxy of single isochrone and have demonstrated that the eMSTO is not a peculiarity of MC clusters (Marino et al. 2018b). Spectroscopy of MS stars in NGC 6705 shows that the blue and the red MS are populated by slow and fast rotators, respectively (Marino et al. 2018b). Similarly, the color and magnitude of eMSTO stars of NGC 2818 and NGC 6705 are connected with their rotational velocity (Bastian et al. 2018; Marino et al. 2018b). These results suggest that rotation plays an important role in shaping eMSTOs and broadened or split MSs in Galactic open clusters, resembling MC clusters.

In this work, we exploit the Gaia data release 2 (DR2; Gaia Collaboration et al. 2018) to analyze photometry, parallaxes, 
and proper motions of a large sample of Galactic open clusters younger than $\sim 2 \mathrm{Gyr}$ to investigate the occurrence of the eMSTO in their CMDs. The paper is organized as follows. In Section 2, we describe the data set and the data analysis. The CMDs and the investigation of the presence of eMSTO and broadened MSs are discussed in Section 3. Section 4 presents a comparison of the data with theoretical models, while Section 5 presents a summary and brief discussion of our results.

\section{Data and Data Analysis}

To unambiguously identify multiple populations along the CMD, if present, we need densely-populated clusters with low differential reddening and negligible contamination from field stars. To do this, we selected all the Galactic open clusters of the new general (NGC), Index (IC), Melotte, and Collinder catalogs that, according to Dias et al. (2002), have $E(B$ $-V)<0.35$ and host more than 400 cluster members. Moreover, we restrict our analysis to clusters older than 2.5 Gyr, as there is no evidence of eMSTO and split MS in MC clusters with similar ages. Our sample also includes NGC 6705 (M11, $E(B-V)=0.43$ ), which exceeds our reddening constraint, because previous evidence of eMSTOs and broadened MS has been reported for this cluster (Marino et al. 2018b).

We downloaded Gaia DR2 astrometry, photometry, parallaxes, and proper motions of stars within a radial distance from the center of each cluster smaller than 2.5 times the cluster radius provided by Dias et al. (2002) and identified a sample of cluster members by using the following iterative procedure, which is illustrated in Figure 1 for NGC 2099.

1. We first analyze the vector-point diagram (VPD) of stellar proper motions, and find that NGC 2099 cluster members are clearly clustered around $\left(\mu_{\alpha} \cos \delta\right.$ : $\left.\mu_{\delta}\right) \sim(1.9$ : -5.6$)$. Hence, we draw by eye a circle in the VPD that encloses most cluster members. The stars within the circle are selected to calculate the median values of $\mu_{\alpha} \cos \delta$ and $\mu_{\delta} \quad\left(\left\langle\mu_{\alpha} \cos \delta\right\rangle\right.$ and $\left.\left\langle\mu_{\delta}\right\rangle\right)$ and to derive the quantity $\mu_{\mathrm{R}}=$ $\left.\sqrt{\left(\mu_{\alpha} \cos \delta-\left\langle\mu_{\alpha} \cos \delta\right\rangle\right)^{2}+\left(\mu_{\delta}-\left\langle\mu_{\delta}\right\rangle\right)^{2}}\right)$.

2. We plotted $G_{\mathrm{RP}}$ as a function of $\mu_{\mathrm{R}}$ for the selected stars and divided the analyzed magnitude interval with $10.0<G_{\mathrm{RP}}<17.5$ into bins of $0.5 \mathrm{mag}$ each. For each bin, we iterativelly calculated the median value of $\mu_{\mathrm{R}}$ $\left(\mu_{\mathrm{R}, \mathrm{med}}\right)$ and the corresponding rms $(\sigma)$ by rejecting all the stars with $\mu_{\mathrm{R}}>\mu_{\mathrm{R} \text {,med }}+4 \cdot \sigma$. The mean magnitudes of each bin are associated to the quantities $\mu_{\mathrm{R} \text {,med }}+4 \cdot \sigma$ and these points are linearly interpolated to derive the orange line plotted in the upper-left panel of Figure 1. Stars with deviations from $\mu_{\mathrm{R} \text {,med }}$ larger than $4 \cdot \sigma$ are excluded from the sample of probable cluster members.

3. We plotted $G_{\mathrm{RP}}$ as a function of the parallax, $\pi$, for the probable cluster members and calculated, for each bin of magnitude defined above, the median parallax $\pi_{\text {med }}$ and the corresponding rms, $\sigma$, by using the same procedure described for proper motions. The orange lines plotted in the central-upper panel of Figure 1 are derived by adding $\pm 4 \cdot \sigma$ to $\pi_{\text {med }}$ and all the stars that lie outside these two lines are excluded from the sample of probable cluster members.

4. The selected stars are used to derive improved estimates of $\left\langle\mu_{\alpha} \cos \delta\right\rangle$ and $\left\langle\mu_{\delta}\right\rangle$. This ends one iteration. The procedure required three or four iterations to reach the convergence.

Photometry of cluster members has been corrected for differential reddening by using the method described by Milone et al. (2012, see their Section 1) and illustrated in Figure 2. Briefly, we first defined the reddening direction by using the absorption coefficients in the $G_{\mathrm{BP}}$ and $G_{\mathrm{RP}}$ bands provided by Casagrande \& VandenBerg (2018). Then, we derived the fiducial of MS stars and calculated the color residuals from this fiducial. To estimate the differential reddening suffered by each star in the analyzed field of view, we selected a sample of 35 neighbors formed by bright MS cluster members that are not evident binaries. Our best differential-reddening estimate corresponds to the median of the color residuals, calculated along the reddening line. To derive the corresponding error, we subtracted the median from the residual of each star and calculated the 68.27th percentile of the distribution of the corresponding absolute values $(\sigma)$. We considered the quantity $1.253 \cdot \sigma / \sqrt{35}$ as the uncertainty associated to the differentialreddening.

As an example, we compare in Figure 2 the original CMD of NGC 2099 cluster members (upper-left panel) with the CMD corrected for differential reddening (upper-right panel). We also plot the differential-reddening map for a circular region with radius of 40 arcmin centered on NGC 2099 (bottom left). The bottom-right panel shows the reddening variation as a function of the relative right-ascension distance from the cluster center for stars in six decl. intervals.

A visual inspection of the differential-reddening corrected CMDs reveals that at least 12 open clusters, namely IC 2714, Melotte 71, NGC 1245, NGC 1817, NGC 2099, NGC 2360, NGC 2818, NGC 3114, NGC 3532, NGC 5822, and NGC 6705, clearly exhibit multiple sequences in their CMDs. Their CMDs are presented and analyzed in the next section.

\section{Multiple Populations Along the CMDs}

The final CMDs, corrected for differential reddening, of the selected cluster members are plotted in Figures 3-4, where we also represent with red error bars the typical observational uncertainties for stars with different luminosities. A visual inspection of these figures clearly reveals that IC 2714, Melotte 71, NGC 1245, NGC 1817, NGC 2099, NGC 2360, NGC 2818, NGC 3114, NGC 3532, NGC 5822, and NGC 6705 exhibit the eMSTO. Noticeably, the upper MS of NGC 2099, NGC 2287 (M41), NGC 3114, NGC 3532, and NGC 6705 is broadened, in contrast with the faint MS, which is narrow and well defined. Similarly to what previously observed in MCs clusters, the broadened MS seems to disappear at the luminosity of the MS kink at $T_{\text {eff }} \sim 7000 \mathrm{~K}$, which is a feature of the CMDs that indicates the onset on envelope convection due to the lowering of the adiabatic gradient in the region of partial hydrogen ionization (e.g., D'Antona et al. 2002).

The eMSTOs and the broadened bright MSs are highlighted in the insets of Figures 3-4. In the following, we demonstrate that they are intrinsic features of the cluster CMDs. To this aim, we investigate the impact of observational uncertainties, residual field-stars contamination and binaries on the appearance of eMSTOs and the broadened MSs on the CMDs.

Specifically, in Section 3.1 we describe the method used to statistically subtract field stars with cluster-like parallaxes and proper motions from the CMDs of candidate cluster members. 

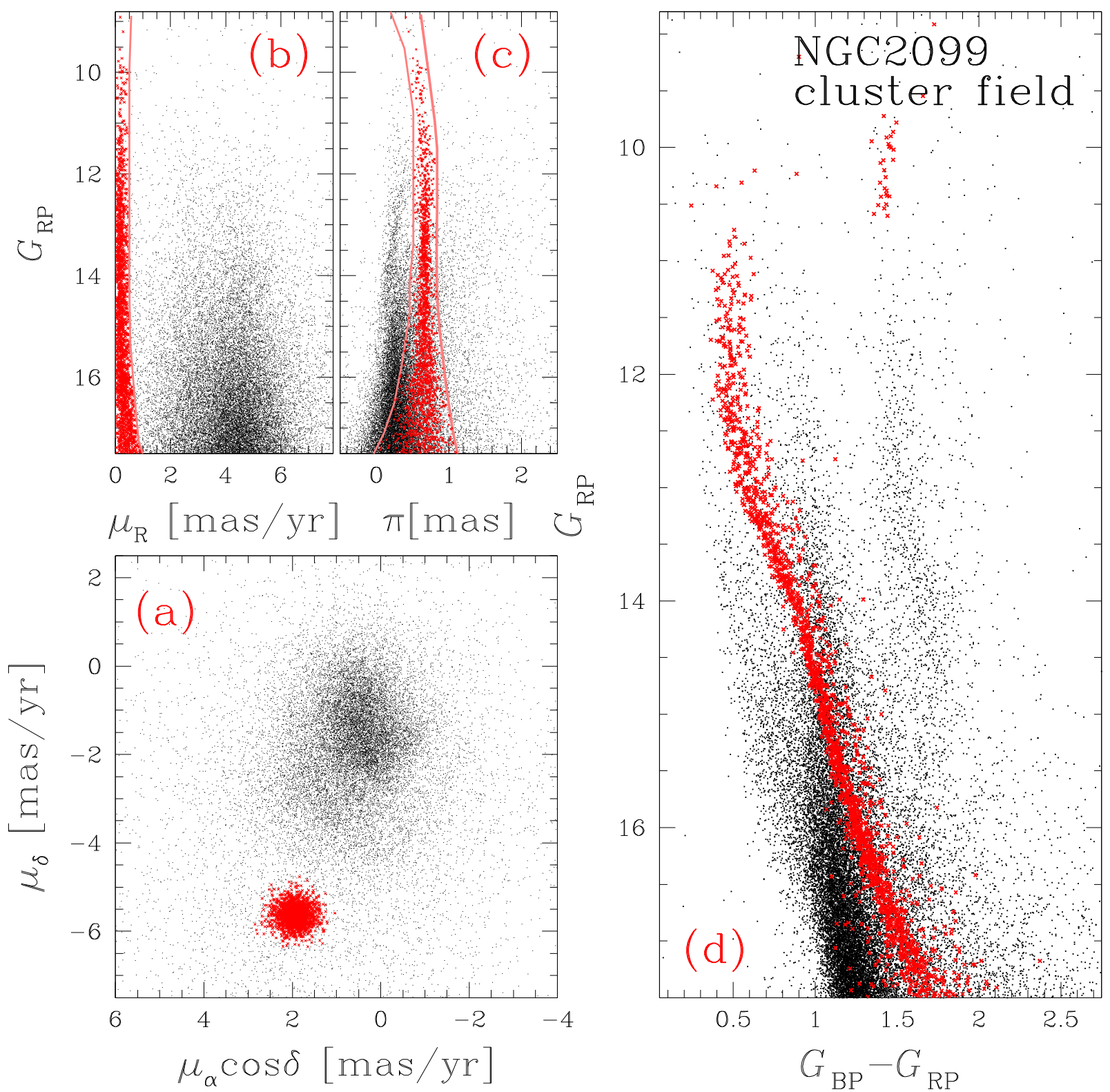

Figure 1. This figure illustrates the procedure that we used to select probable members of NGC 2099. The VPD of proper motions for stars in the cluster field is plotted in panel (a), while panels (b) and (c) show $G_{\mathrm{RP}}$ against proper motions and parallaxes, respectively. The red lines are used to separate NGC 2099 members from field stars. The $G_{\mathrm{RP}}$ vs. $G_{\mathrm{BP}}-G_{\mathrm{RP}}$ CMD is illustrated in panel (d). Selected cluster members are represented with red symbols. See the text for details.

In Section 3.2, we estimate the fraction of binaries in each cluster, and in Section 3.3 we compare the observations with simulated CMDs that account for both binaries and observational errors.

\subsection{Field Stars}

The CMDs shown in Figures 3-4 are mostly populated by cluster members that have been selected on the basis of their parallaxes and proper motions as described in Section 2. To estimate the contamination from those field stars that have proper motions and distances similar to those of cluster members, we applied the procedure illustrated in Figure 5 for NGC 2099.

All the stars plotted in Figure 5 are located in the "reference field", which is a circular annulus with the same area as the cluster field, centered on the cluster and with internal radius corresponding to three times the cluster radius provided by Dias et al. (2002). The VPD of proper motions for stars in the reference field is plotted in Figure 5(a); panels (b) and (c) show the $G_{\mathrm{RP}}$ magnitude as a function of parallax and proper motions, respectively. We plot in each panel the orange lines derived in Figure 1 that are now used to select field stars with cluster-like proper motions and parallaxes, in close analogy with what we did for candidate cluster members. The stars with cluster-like proper motions have been selected according to their position in the diagrams plotted in panels (b) and (c) and are represented with aqua crosses in all the panels of 5 .

To statistically subtract the selected field stars from the cluster-field CMD, we adopted the same procedure used in our previous papers (e.g., Milone et al. 2009). In a nutshell, we calculated for each selected star (i) in the reference field a distance in the CMD $d_{\mathrm{i}}=\sqrt{\begin{array}{l}k\left(\left(G_{\mathrm{BP}, \mathrm{rf}}^{\mathrm{i}}-G_{\mathrm{RP}, \mathrm{rf}}^{\mathrm{i}}\right)-\left(G_{\mathrm{BP}, \mathrm{cf}}-G_{\mathrm{RP}, \mathrm{cf}}\right)\right)^{2} \\ +\left(G_{\mathrm{RP}, \mathrm{rf}}^{\mathrm{i}}-G_{\mathrm{RP}, \mathrm{cf}}\right)^{2}\end{array} \quad \text { where }}$ 

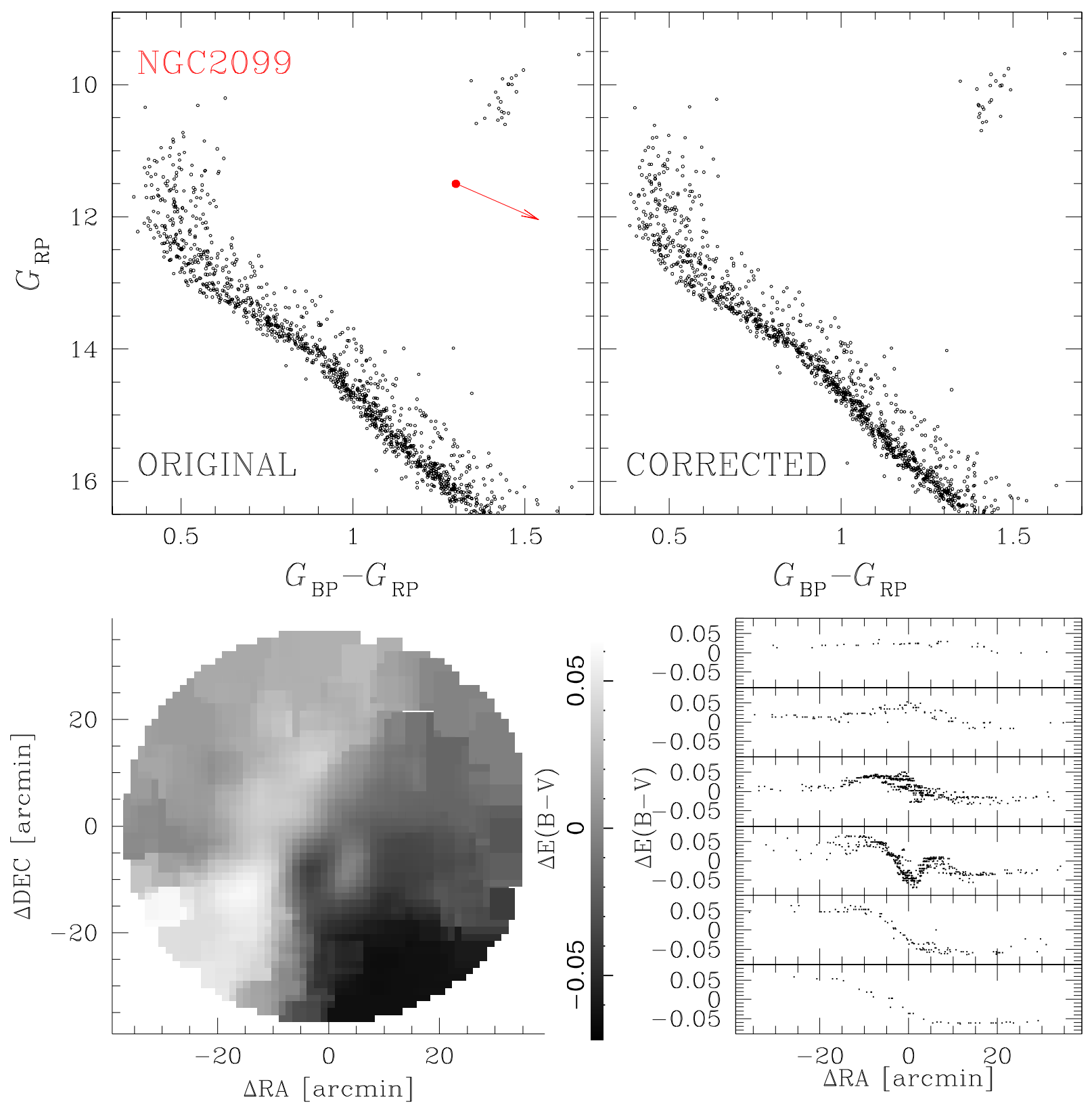

Figure 2. Upper panels: comparison of the original CMD of selected cluster members of NGC 2099 (left) with the CMD of the same stars corrected for differential reddening (right). The arrow plotted in the left-panel CMD indicates the reddening vector and corresponds to $\Delta E(B-V)=0.3$ mag. Lower panels: map of differential reddening, centered on NGC 2099. The levels of gray correspond to different $E(B-V)$ values as indicated by the scale on the middle (left). Right panels show $E(B-V)$ as a function of the right-ascension distance from the cluster center for stars in six slices of decl.

$G_{\mathrm{BP}, \mathrm{rf}(\mathrm{cf})}$ and $G_{\mathrm{RP}, \mathrm{rf}(\mathrm{cf})}$ are the magnitudes of the selected stars in the reference (cluster) field, and $k=7$ is a constant that accounts for the fact that the color of a star is better constrained than its magnitude (Gallart et al. 2003; Marino et al. 2014). We finally subtracted the stars in the cluster-field CMD with the smallest distance.

\subsection{Binaries}

Unresolved binaries formed by pairs of MS stars are redder and brighter than single MS stars with similar masses while binaries formed by an MSTO star and an MS or an MSTO star are brighter than the corresponding single MSTO stars. In the following, we measure the fraction of MS-MS binary systems of each cluster to estimate the contribution of binaries to the eMSTO and the broadened MS.
To estimate the fraction of unresolved binaries with $q>0.7$, we used the procedure illustrated in Figure 6 for NGC 2099, which is based on the method by Milone et al. $(2012,2016 \mathrm{a})$ to characterize binaries in Galactic GCs. We first identified two points along the MS with magnitudes $G_{\mathrm{RP}}^{\text {bright }}$ and $G_{\mathrm{RP}}^{\text {faint }}$, that delimit the MS region where the high-mass binaries are clearly separated from the remaining MS stars and there is no evidence for broadened or split sequences. We then defined two regions in the $\mathrm{CMD}$, namely $\mathrm{A}$ and $\mathrm{B}$, that correspond to the gray shaded areas in the CMDs of Figure 6: region A includes all the single stars with $G_{\mathrm{RP}}^{\text {bright }}<G_{\mathrm{RP}}<G_{\mathrm{RP}}^{\text {faint }}$ and all the binaries with a primary component in the same magnitude interval; region $\mathrm{B}$ is the sub-region of $\mathrm{A}$ that is populated by binaries with $q>0.7$ and is represented with dark-gray colors in Figure 6. The reddest line plotted in Figure 6 is the fiducial of 

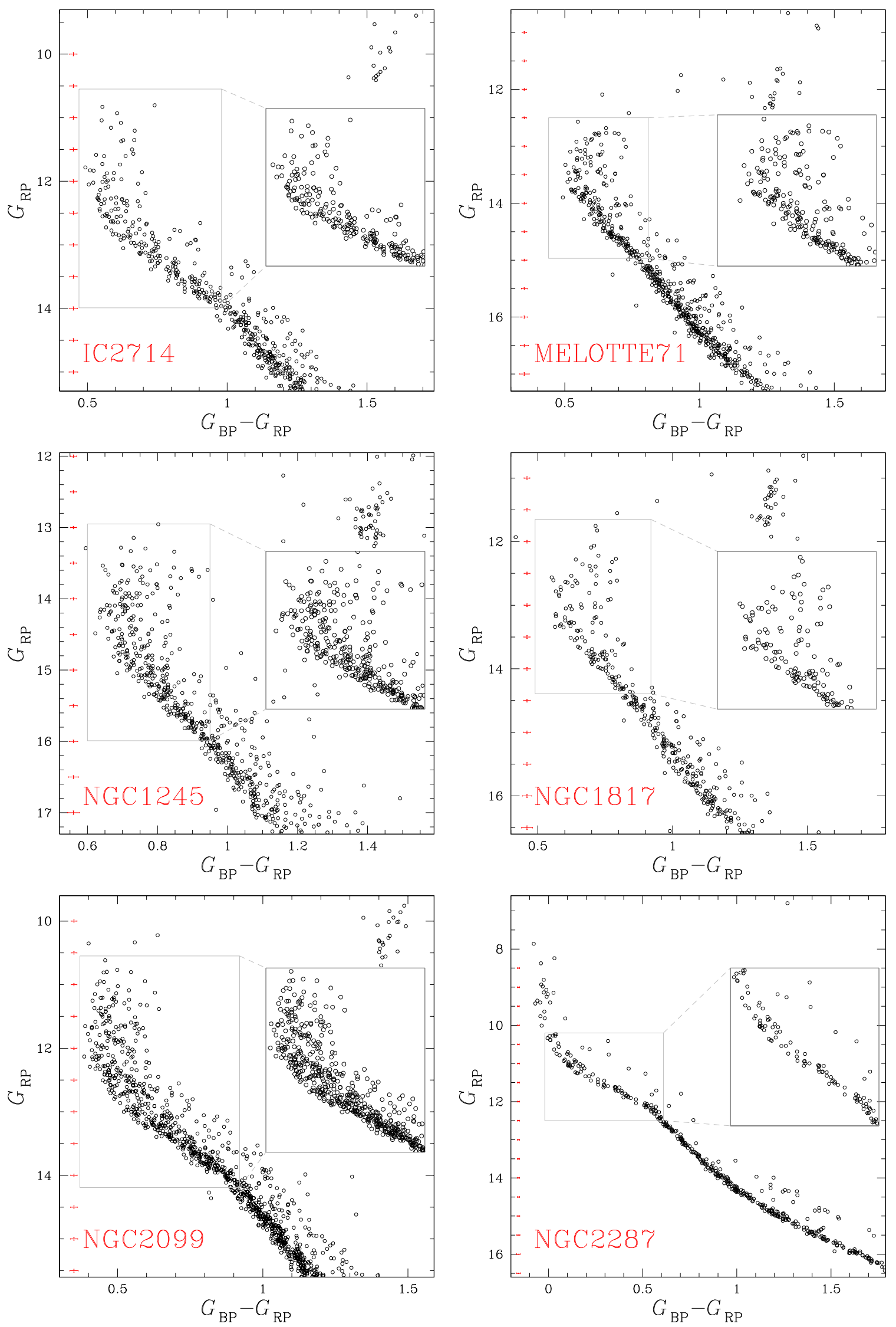

Figure 3. $G_{\mathrm{RP}}$ vs. $G_{\mathrm{BP}}-G_{\mathrm{RP}}$ CMDs, corrected for differential reddening, of cluster members for IC 2714, Melotte 71, NGC 1245, NGC 1817, NGC 2099, and NGC 2287. The insets highlight the eMSTO or the broadened MS. Red bars represent typical observational uncertainties. 

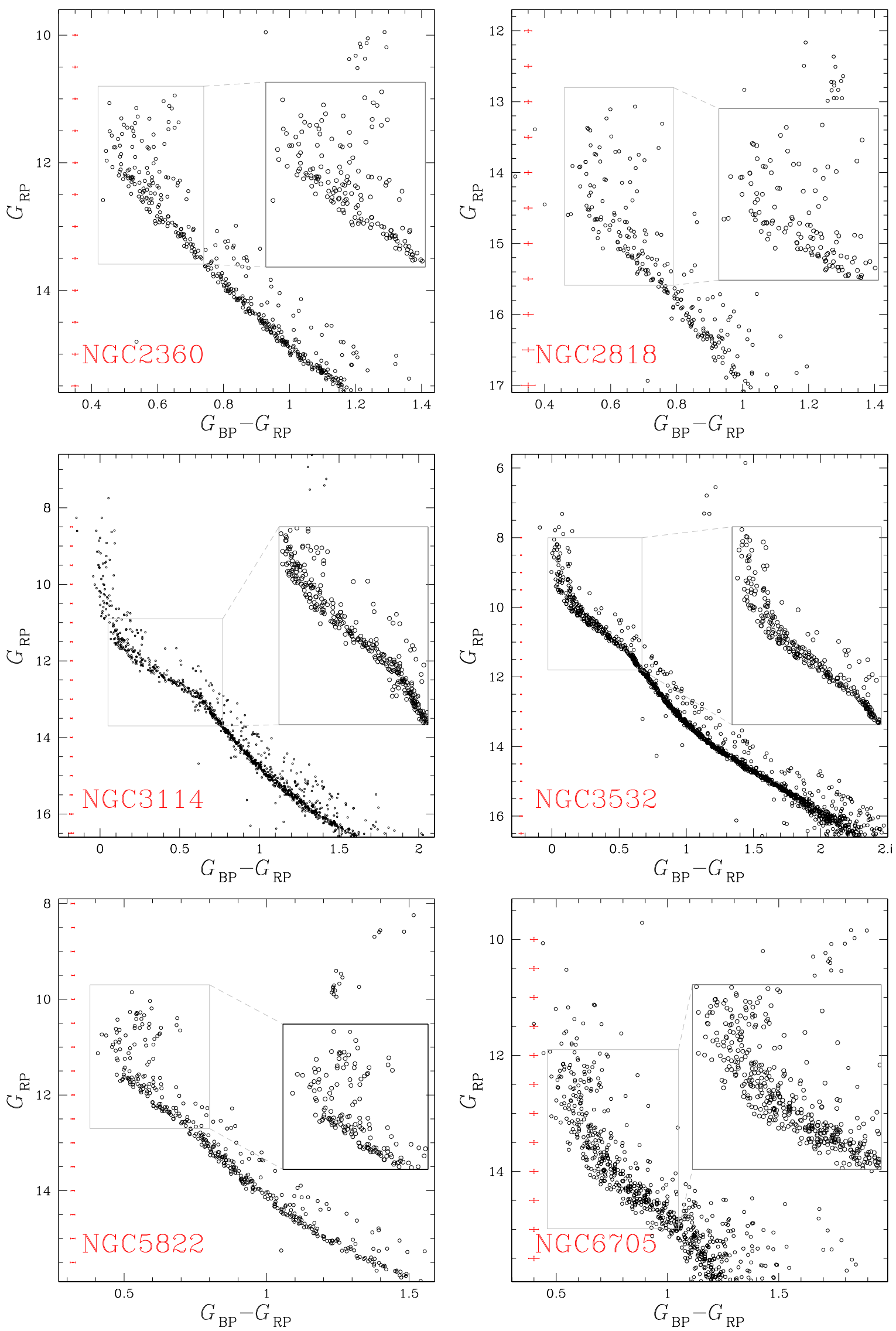

Figure 4. The same as in Figure 3 but for NGC 2360, NGC 2818, NGC 3114, NGC 3532, NGC 5822, and NGC 6705. 

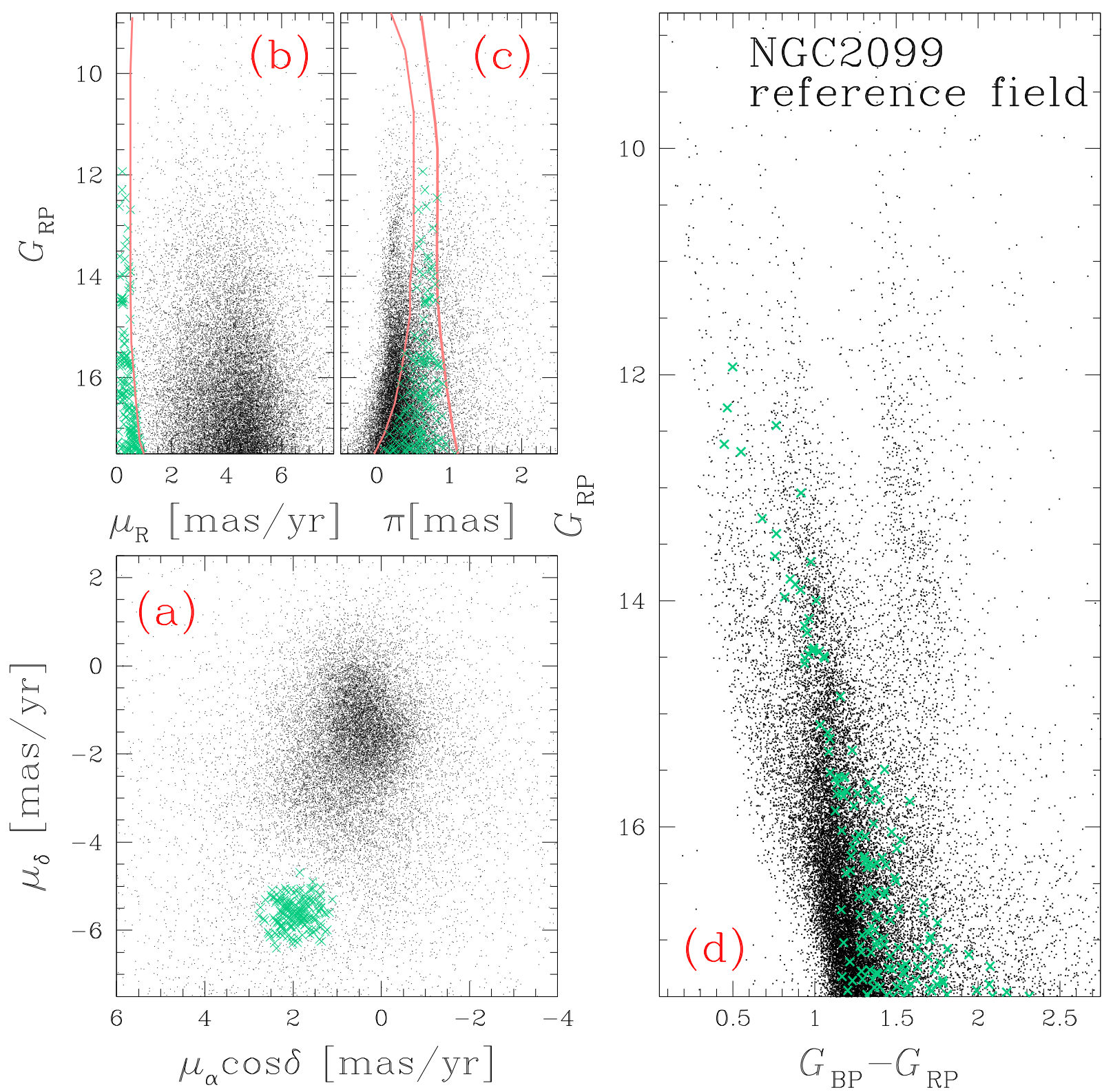

Figure 5. This figure illustrates the procedure that we used to identify field stars with similar proper motions and parallaxes as NGC 2099 cluster members. The VPD of proper motions of stars in the "reference field" is plotted in panel (a), while panels (b) and (c) show $G_{\mathrm{RP}}$ as a function of stellar proper motions and parallaxes, respectively. The orange lines defined in Figure 1 are overimposed to the diagrams of panels (b) and (c). The $G_{\mathrm{RP}}$ vs. $G_{\mathrm{BP}}-G_{\mathrm{RP}} \mathrm{CMD}$ is illustrated in panel (d). Selected field stars are marked with aqua crosses. See the text for details.

equal-mass binaries shifted by four times the observational error in color to the red and the bluest line is the MS fiducial line shifted by four times the error in color to the blue. The fiducial of binaries with $q=0.7$ is represented by the blue continuous line and is derived by using the mass-luminosity relation inferred from the best-fit isochrone from Marigo et al. (2017). For each cluster, we assumed the metallicity provided by Paunzen et al. (2010), while the adopted values of ages, reddening and distance modulus are those providing the best match between the data and the isochrones and are listed in Table 1.

The fraction of binaries is calculated as

$$
f_{\mathrm{bin}}^{q>0.7}=\frac{N_{\mathrm{cl}}^{\mathrm{B}}-N_{\mathrm{fi}}^{\mathrm{B}}}{N_{\mathrm{cl}}^{\mathrm{A}}-N_{\mathrm{fi}}^{\mathrm{A}}}-\frac{N_{\mathrm{sim}}^{\mathrm{B}}}{N_{\mathrm{sim}}^{\mathrm{A}}}
$$

where $N_{\mathrm{cl}}^{\mathrm{A}(\mathrm{B})}$ is the number of cluster members in the region A (B) of the $\mathrm{CMD}, N_{\mathrm{fi}}^{\mathrm{A},(\mathrm{B})}$ and $N_{\mathrm{sim}}^{\mathrm{A},(\mathrm{B})}$ are the corresponding numbers of field stars with cluster-like proper motions and parallaxes and the number of simulated stars, respectively.

The measured fraction of binaries with $\mathrm{q}>0.7, f_{\mathrm{bin}}^{q}>0.7$ is used to extrapolate the total fraction of binaries, $f_{\text {bin }}^{\text {TOT }}$. Specifically, by assuming a flat mass-ratio distribution, as observed among binaries with $\mathrm{q}>0.5$ of Galactic GCs (Milone et al. 2012, 2016b), we infer $f_{\text {bin }}^{\text {TOT }} \sim 3.3 f_{\text {bin }}^{q>0.7}$. The total fraction binaries is typically around 0.30 and ranges from $\sim 0.11$ for NGC 2287 to $\sim 0.51$ for NGC 6705 and is similar to that observed in LMC clusters with similar ages (Milone et al. 2009, see their Table 2). We thus confirm previous findings that open clusters typically host larger binary fraction than Galactic Globular Clusters (e.g., Sollima et al. 2010). 


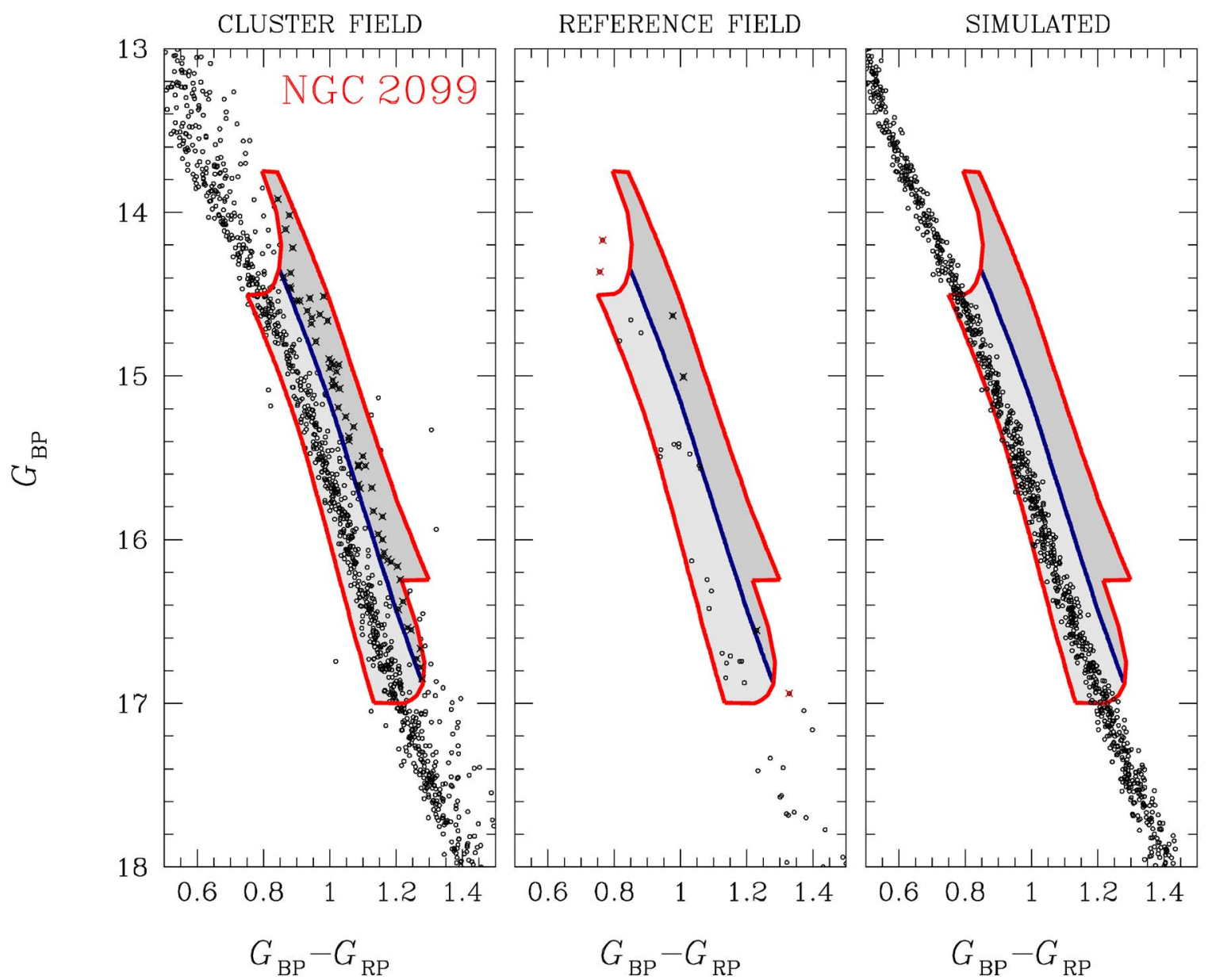

Figure 6. $G_{\mathrm{BP}}$ vs. $G_{\mathrm{BP}}-G_{\mathrm{RP}}$ CMD of selected NGC 2099 cluster members in the cluster field (left panel) and CMD of stars with cluster-like proper motions and parallaxes in the reference field (middle panel). Right panel shows the simulated CMD. The shaded areas indicate the region A of the CMD, which is populated single MS stars and by MS-MS binary pairs with a primary component in the mass interval between 1.06 and 1.63 solar masses. The blue lines represent the fiducial lines of binaries with mass ratio, $q=0.7$. The region $\mathrm{B}$ of the $\mathrm{CMD}$, which is populated by binaries with $q \geqslant 0.7$ (black crosses), is colored dark-gray. See the text for details.

Table 1

Distance Modulus, Reddening, Age, FWHM of the Age Distribution, Fraction of Binaries with Mass-ratio $q>0.7$, and Total Fraction of Binaries Inferred in This Paper

\begin{tabular}{|c|c|c|c|c|c|c|c|}
\hline Cluster & $(m-M)_{0}$ & $E(B-V)$ & $Z$ & Age (Myr) & FWHM (Myr) & $f_{\text {bin }}^{q>0.7}$ & $f_{\text {bin }}^{\text {tot }}$ \\
\hline IC 2714 & 10.60 & 0.38 & 0.0205 & 540 & $134 \pm 57$ & 0.105 & 0.350 \\
\hline MELOTTE 71 & 11.60 & 0.22 & 0.0095 & 1220 & $165 \pm 38$ & 0.085 & 0.283 \\
\hline NGC 1245 & 12.45 & 0.29 & 0.0183 & 1000 & $139 \pm 21$ & 0.119 & 0.397 \\
\hline NGC 1817 & 11.00 & 0.26 & 0.0100 & 1030 & $165 \pm 47$ & 0.083 & 0.277 \\
\hline NGC 2099 & 10.90 & 0.26 & 0.0300 & 580 & $125 \pm 21$ & 0.085 & 0.283 \\
\hline NGC 2287 & 9.40 & 0.04 & 0.0219 & 280 & $76 \pm 28$ & 0.034 & 0.113 \\
\hline NGC 2360 & 10.23 & 0.16 & 0.0140 & 1020 & $210 \pm 35$ & 0.087 & 0.290 \\
\hline NGC 2818 & 12.35 & 0.22 & 0.0100 & 1110 & $160 \pm 59$ & 0.088 & 0.293 \\
\hline NGC 3114 & 10.05 & 0.12 & 0.0209 & 180 & $75 \pm 31$ & 0.068 & 0.227 \\
\hline NGC 3532 & 8.30 & 0.06 & 0.0160 & 430 & $140 \pm 50$ & 0.074 & 0.247 \\
\hline NGC 5822 & 9.40 & 0.11 & 0.0170 & 1130 & $270 \pm 52$ & 0.131 & 0.437 \\
\hline NGC 6705 & 11.10 & 0.46 & 0.0083 & 570 & $245 \pm 71$ & 0.153 & 0.510 \\
\hline
\end{tabular}

Note. Cluster metallicities are from Paunzen et al. (2010).

\subsection{Simulated CMDs}

The obtained total binary fractions, listed in Table 1, are used to simulate the CMD of a simple stellar population with the same observational errors, age, metallicity, distance modulus and reddening as inferred from the observations. To do this, we first associated a synthetic star to each star in the observed CMD of cluster member, with the same magnitude and the color of the fiducial line. We selected a fraction of single stars equal to $f_{\text {bin }}^{\text {TOT }}$ and estimated the mass $\mathcal{M}$ of each of them by using the mass-luminosity relation by Marigo et al. (2017). We 

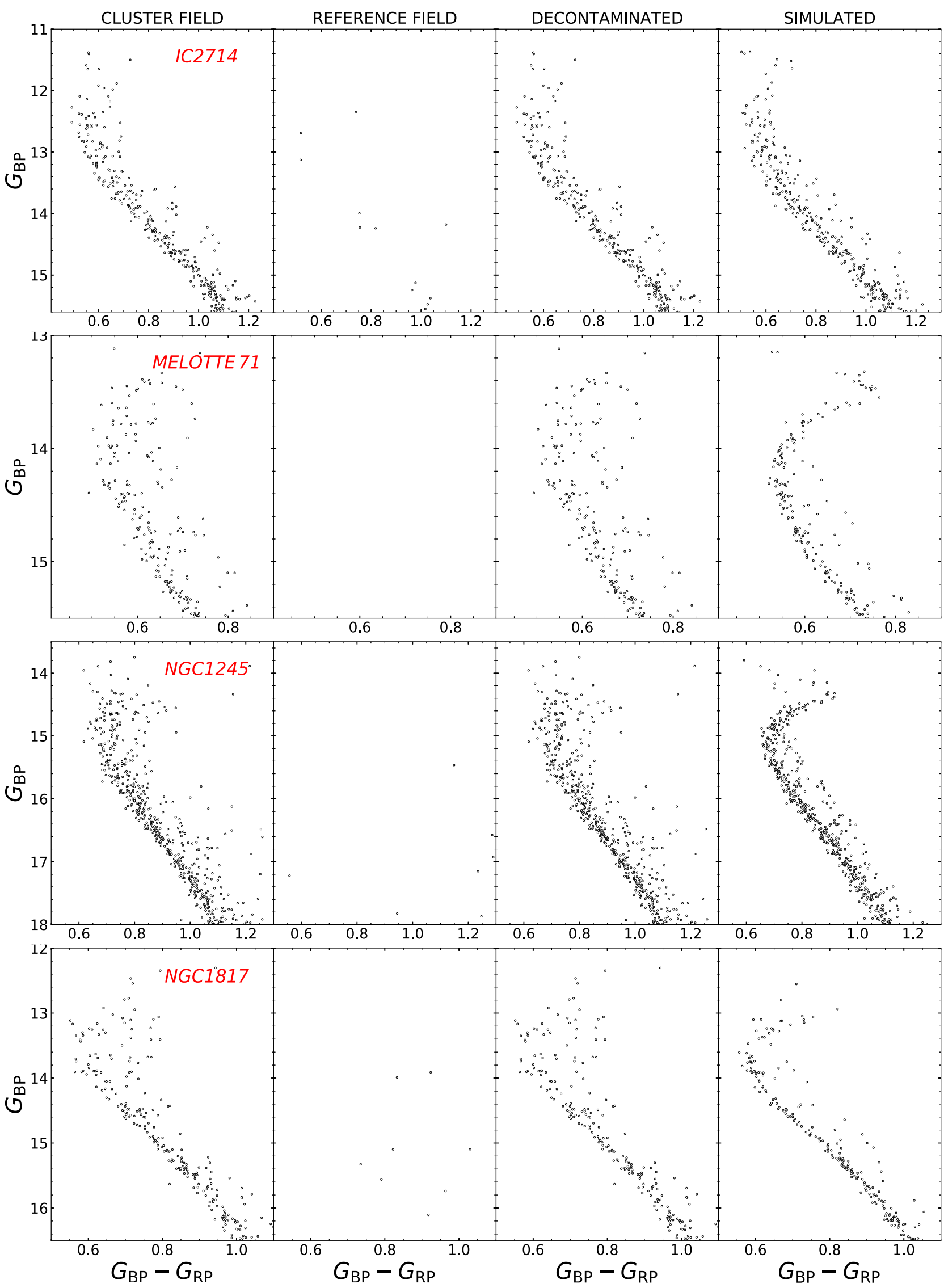

Figure 7. From the left to the right. CMD of the selected cluster members in the cluster field for IC 2714, Melotte 71, NGC 1245, and NGC 1817 (first column), CMD of reference-field stars with cluster-like parallaxes and proper motions (second column), CMD of cluster members after the statistical subtraction of field stars with cluster-like parallaxes and proper motions (third column). Simulated CMD (forth column). 

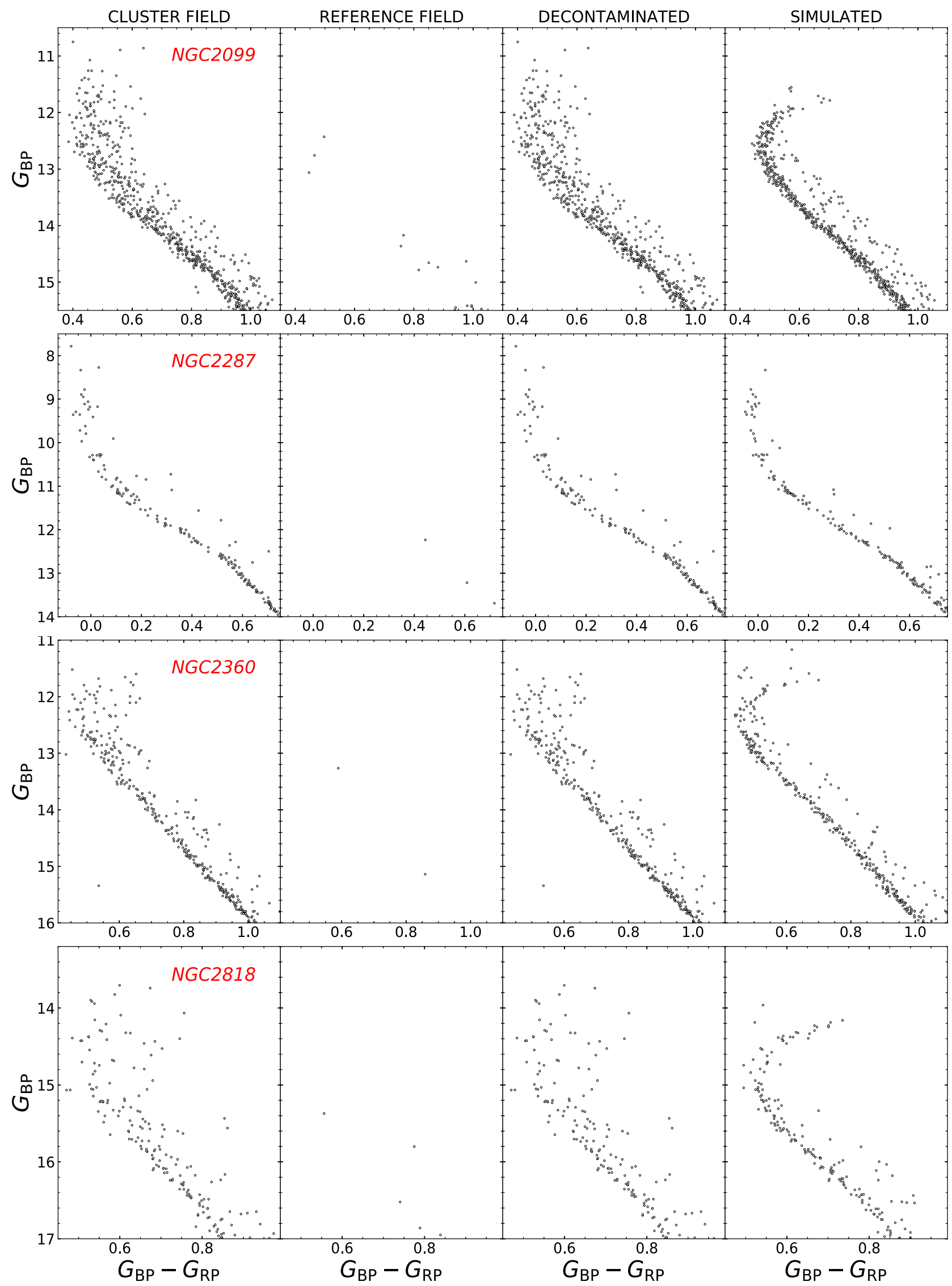

Figure 8. The same as in Figure 7 but for NGC 2099, NGC 2287, NGC 2360, and NGC 2818. 

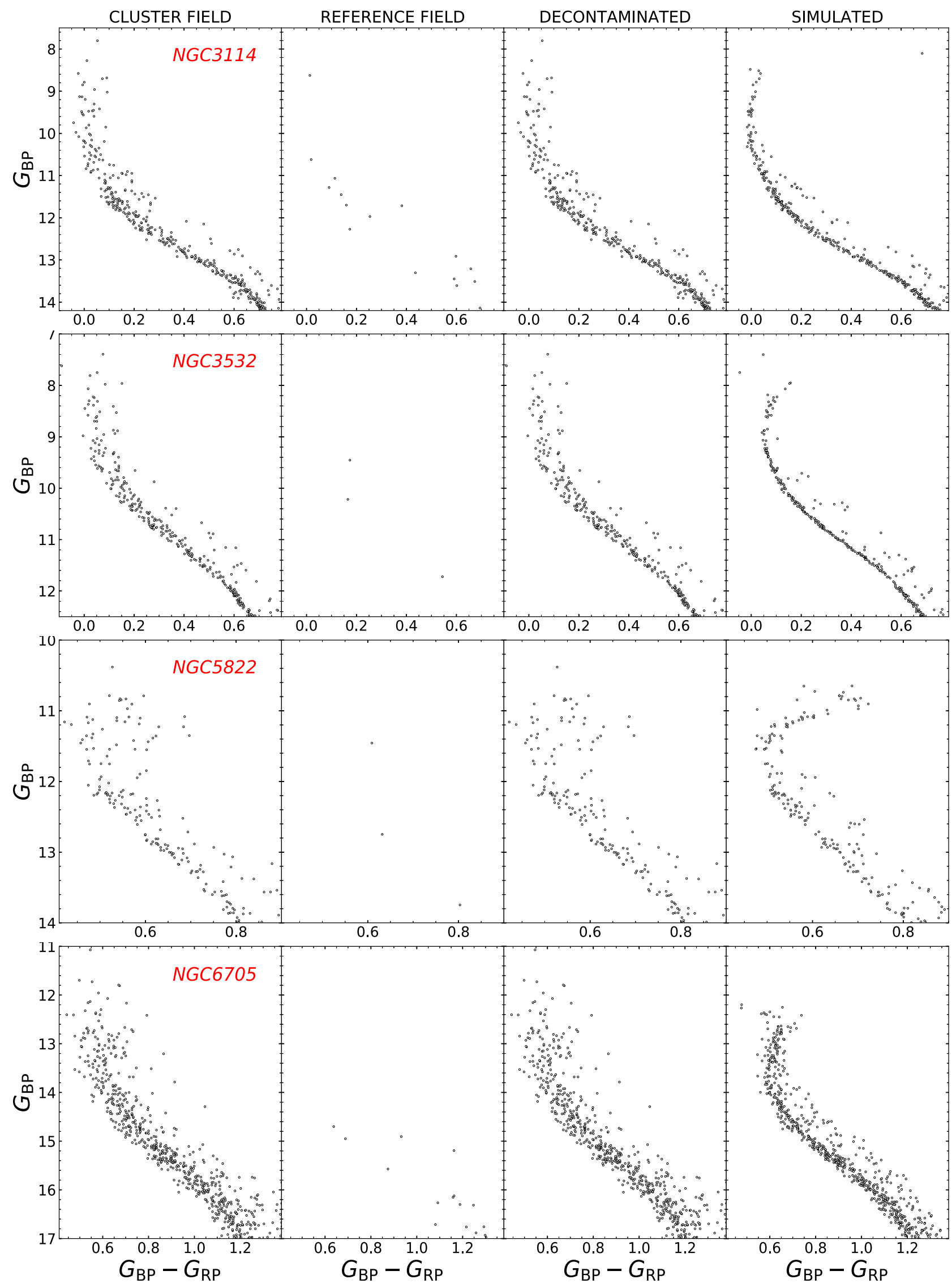

Figure 9. The same as in Figure 7 but for NGC 3114, NGC 3532, NGC 5822, and NGC 6705. 


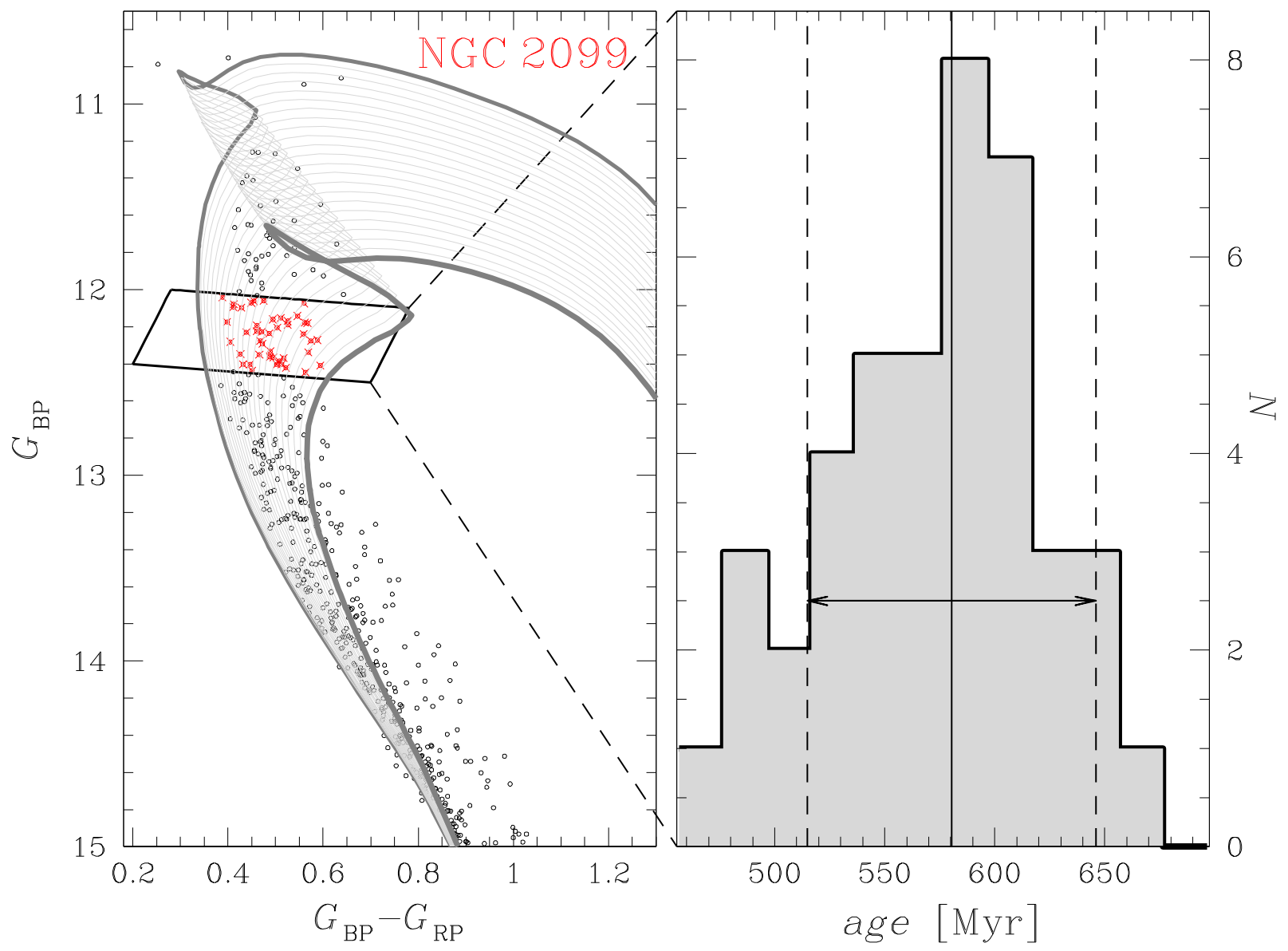

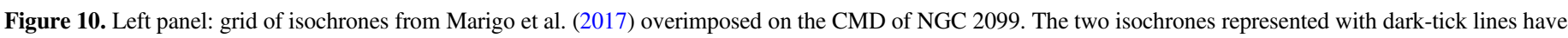

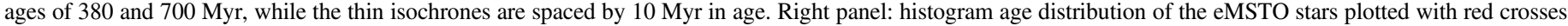

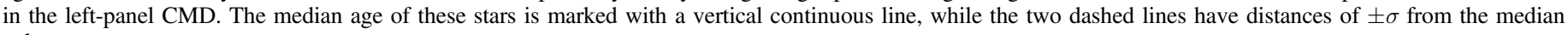
value.

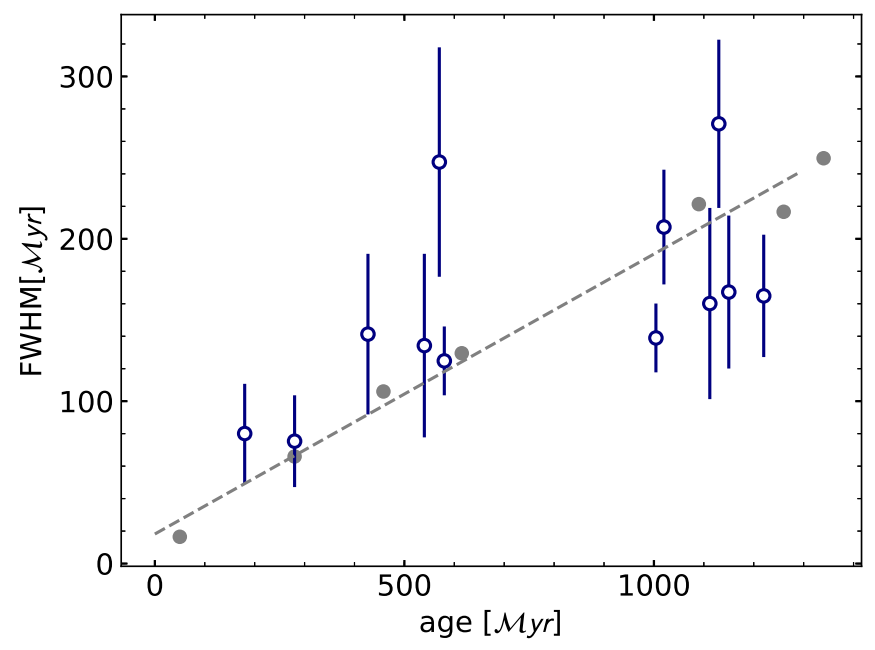

Figure 11. Full width half maximum of the age distribution as a function of cluster age. Blue dots with error bars refer to the analyzed clusters. Gray dots are derived from synthetic CMDs of coeval stellar population with different rotation rates. The dashed line is the least-squares best-fit straight line for the gray dots. See the text for details.

associated a secondary star with a mass $\mathcal{M}_{2}=q \cdot \mathcal{M}$ to each selected star and derived its $G_{\mathrm{RP}}$ magnitude from the relations by Marigo and collaborators. The corresponding color has been inferred from the fiducial line. Finally, we summed up the $G_{\mathrm{BP}}$ and $G_{\mathrm{RP}}$ fluxes of the two components, derived the corresponding magnitudes replaced the original star in the CMD with this binary system, and summed up the observational errors to all the stars of the CMD.

Results are illustrated in Figures 7-9, where we compare for each cluster the CMD of selected cluster members (first column of panels), the CMD with cluster-like proper motions and parallaxes of stars in the reference field (second column), the decontaminated CMD (third column), and the simulated CMDs. A visual inspection of these figures clearly demonstrates that the eMSTOs and the broadened MSs are not due neither to unresolved binaries nor to residual field-star contamination.

\section{Comparison with Theory}

The eMSTOs of Magellanic-Cloud clusters has been interpreted either as the signature of stellar populations with different ages (e.g., Mackey et al. 2008; Goudfrooij et al. 2011) or as the effect of stellar rotation on a single stellar population (e.g., Bastian \& de Mink 2009; Yang et al. 2013; D'Antona et al. 2017; Marino et al. 2018a). To disentangle between these two possibilities, in this section we compare the observed CMDs with isochrones of different ages and with simulated CMDs of stars with different rotation rates.

To estimate the age spread, in the hypothesis that the eMSTO is entirely due to a prolonged star formation, we compared the observed CMDs with isochrones by Marigo et al. 

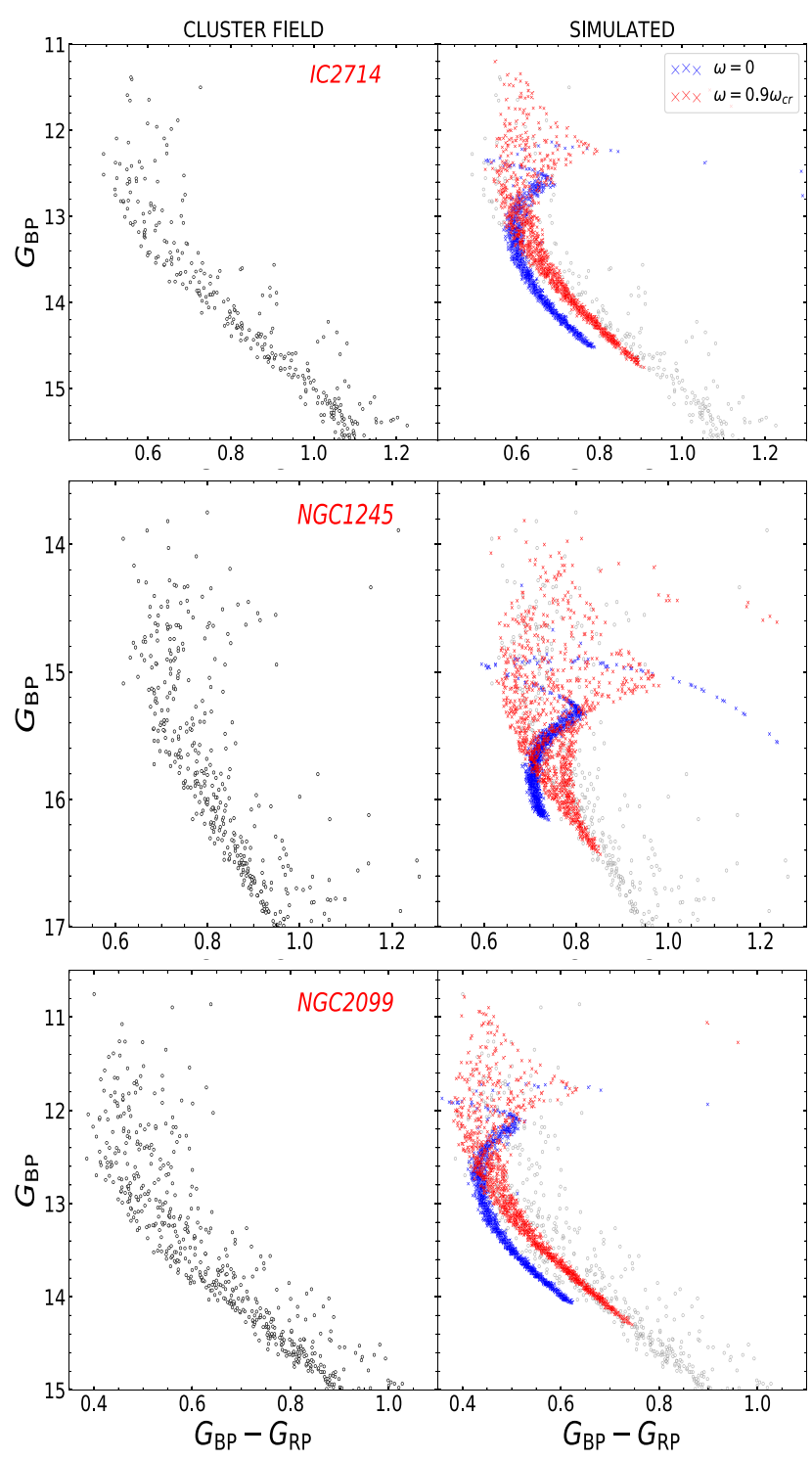
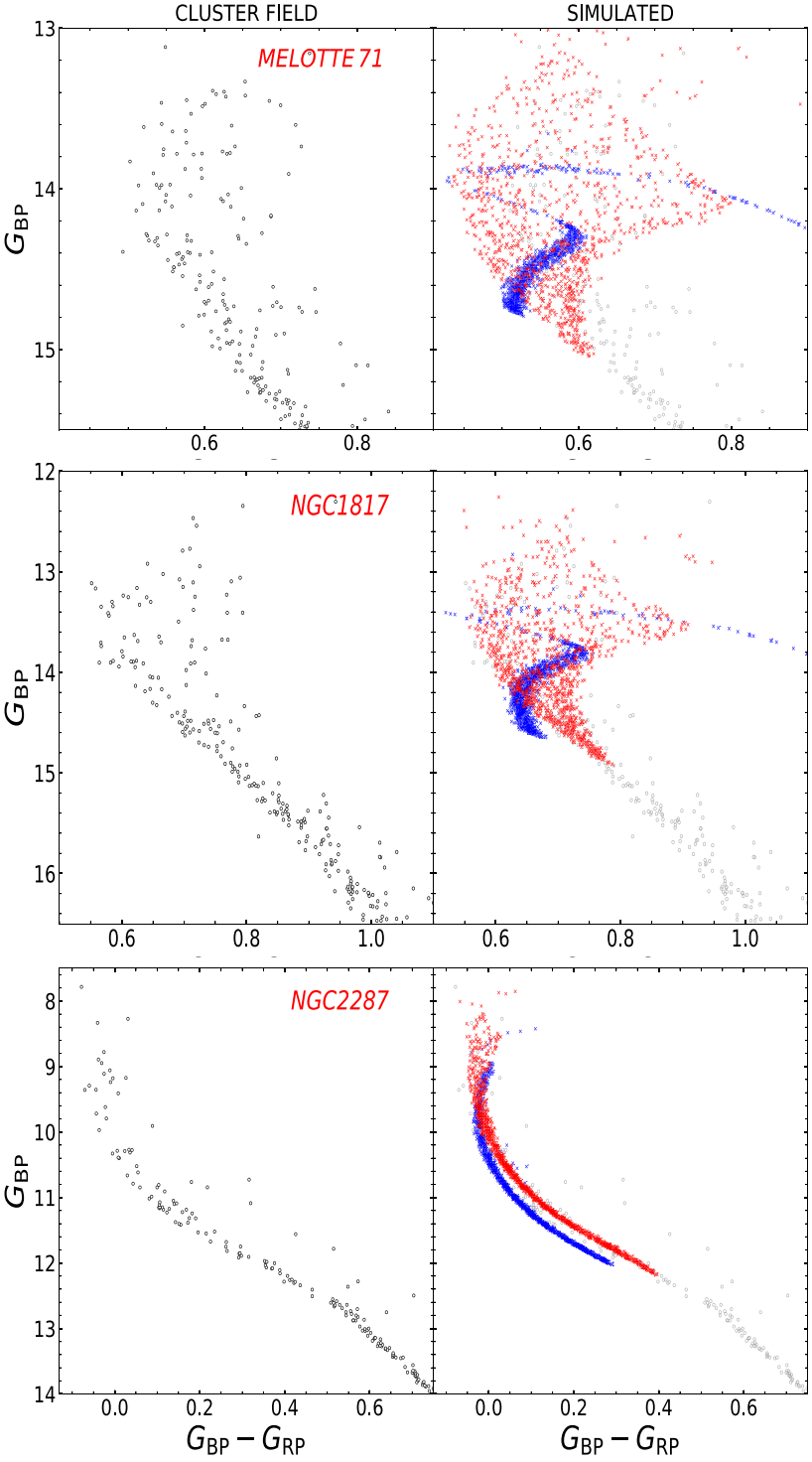

Figure 12. Left panels: reproduction of the observed CMDs of cluster members of IC 2714, Melotte 71, NGC 1245, NGC 1817, NGC 2099 and NGC 2287. Right panels: comparison of the observed CMDs plotted in the left panels (gray dots) and simulated CMDs of a non-rotating stellar population (blue) and of a stellar population with rotation $\omega=0.9 \omega_{\mathrm{cr}}$ (red).

(2017). The procedure that was exploited to derive accurate age distributions is illustrated in Figure 10 for NGC 2099 and is similar to what we have used in previous work (e.g., Milone et al. 2015).

In a nutshell, we first derived by hand the parallelepiped plotted in Figure 10 with the criterion of selecting the region around the turn off where the color and magnitude spread due to age variation are clearly distinguishable. Only stars within the parallelepiped are used to infer the age distribution. Then, we overimposed on the CMD a grid of isochrones with the same metallicity and $[\alpha / \mathrm{Fe}]$ and ages between 380 and $700 \mathrm{Myr}$ in steps of $10 \mathrm{Myr}$ (gray lines in Figure 10) and derived isochrones separated by 1 Myr by linearly interpolating among these isochrones. We associated the age of the closest isochrone to each star and derived the age distribution shown in the right panel of Figure 10. Finally, we calculated the median age and the absolute value of the difference between the age of each star and the median. We considered the 68.27th percentile of the distribution of these absolute values as indicative of the observed age spread, $\sigma_{\mathrm{AGE}, \mathrm{obs}}$. To estimate the contribution of observational errors on the inferred age spread, we applied the procedure described above to the simulated CMD of a simple population and derived the corresponding age spread, $\sigma_{\mathrm{AGE}, \text { sim. }}$. The intrinsic age spread is estimated as $\sigma_{\mathrm{AGE}}=\sqrt{\sigma_{\mathrm{AGE}, \text { obs }}^{2}-\sigma_{\mathrm{AGE}, \text { sim }}^{2}}$. Uncertainties on $\sigma_{\mathrm{AGE}}$ are derived by bootstrapping with replacements performed 1000 times on both the observed and the simulated age distributions.

Our results are summarized in Table 1 where we provide the full width half maximum of the age distribution, $\mathrm{FWHM}=2.355 \cdot \sigma_{\mathrm{AGE}}$, for each cluster. We find that the FWHM ranges from $\sim 70$ for NGC 3114 to $\sim 260 \mathrm{Myr}$ for NGC 5822 and correlates with the cluster age as shown in Figure 11, with old clusters having, on average, larger age spread than younger clusters. A similar trend between the age spread inferred from the eMSTO and the cluster age is also present among MC clusters and is interpreted as the signature of stellar rotation. Indeed, since rotating stars have longer MS lifetime than non-rotating stars with the same age and mass, 

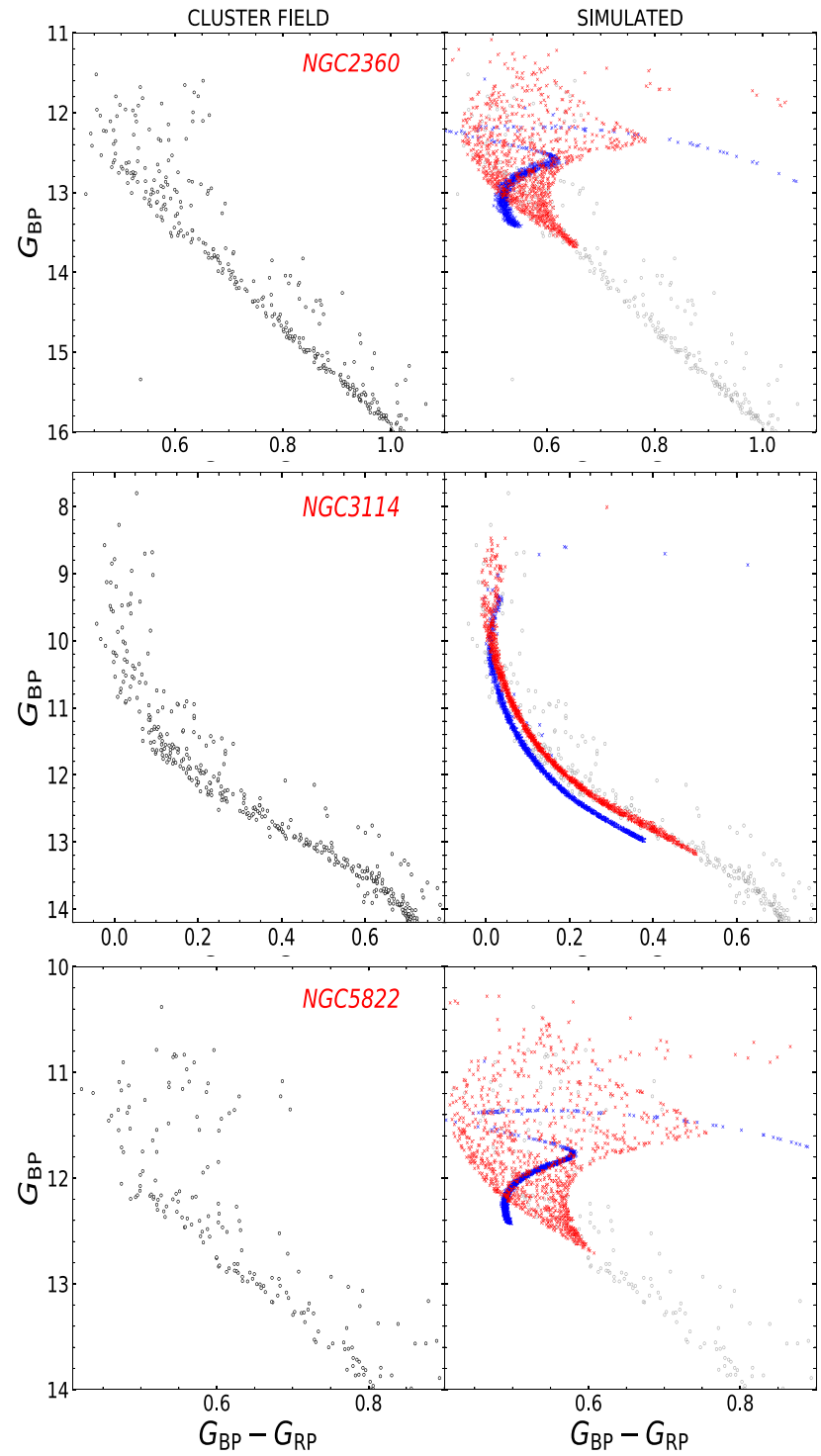
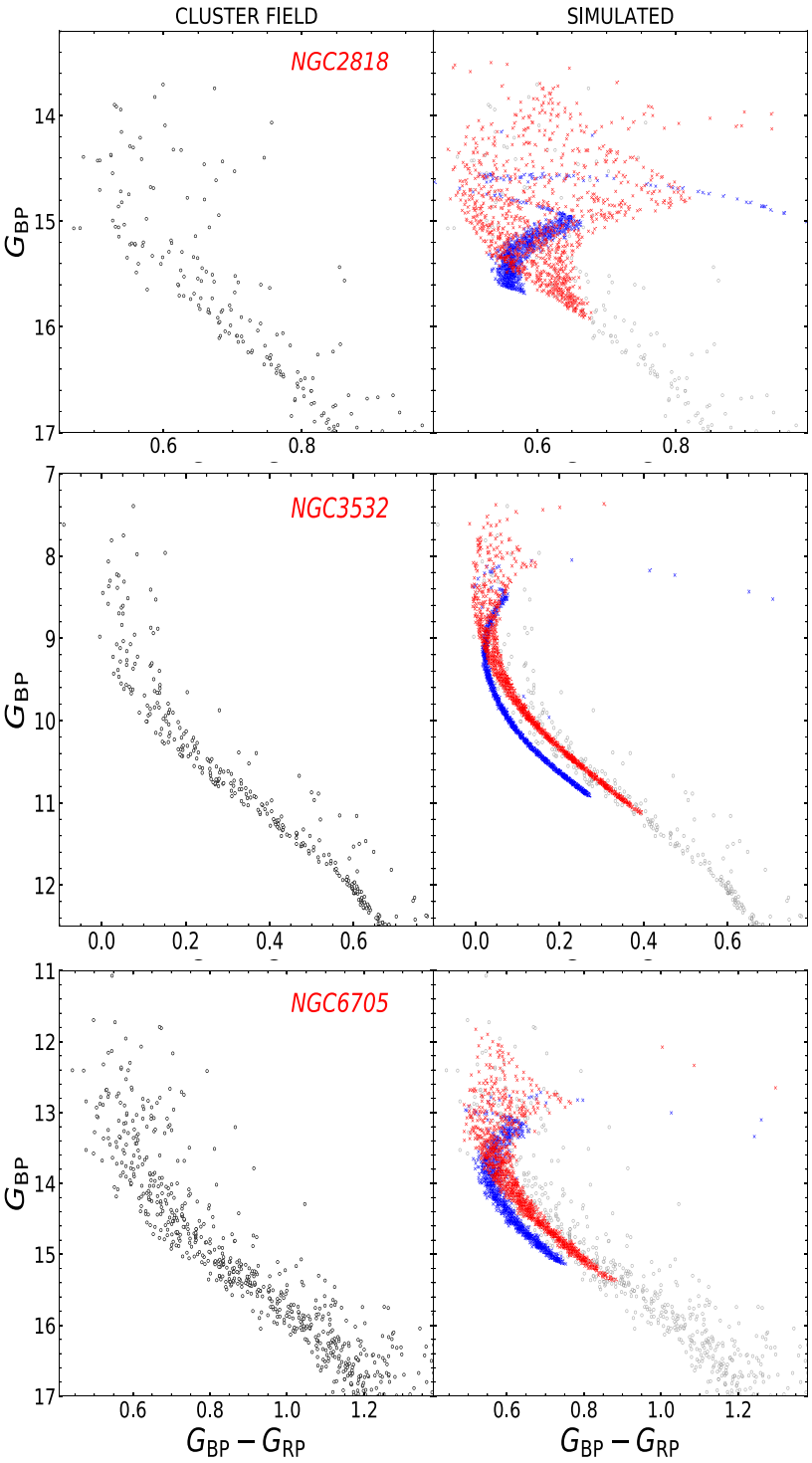

Figure 13. The same as in Figure 12 but for NGC 2360, NGC 2818, NGC 3114, NGC 3532, NGC 5822 , and NGC 6705.

they would appear younger than coeval non-rotating stars within the same cluster. In this case, if the resulting eMSTO is interpreted as an age spread, the resulting age spread would correlate with the cluster age (Niederhofer et al. 2015; Bastian et al. 2018). On the other hand, in the case of a true age spread, we would expect that the amount of age spread does not depend on cluster age, and therefore a correlation would be very unlikely.

To further investigate the effect of rotation on the observed CMDs, we extended the method by Niederhofer and collaborators to Galactic open clusters and compared the observations with simulated CMDs of coeval stellar populations with different rotation rates based on stellar models from the Geneva database with $Z=0.014$ and various ages (Mowlavi et al. 2012; Ekström et al. 2012; Georgy et al. 2014). To simulate the CMDs, we first retrieved the synthetic photometry corresponding to the best-fit non-rotating isochrones, and for the isochrones with rotation equal to 0.9 times the breakout value $\left(\omega=0.9 \omega_{\mathrm{cr}}\right)$. These data account for the limb-darkening effect as in Claret (2000), adopt the gravitydarkening model by Espinosa Lara \& Rieutord (2011), and assume random distribution for the viewing angle. We transformed the synthetic photometry into the observational plane by adopting the model atmospheres by Castelli \& Kurucz (2000) and the transmission curves of the $G_{\mathrm{BP}}$ and $G_{\mathrm{RP}}$ filters of Gaia. We assumed that one third of stars in the simulated CMD do not rotate, while two-thirds of stars have $\omega=0.9 \omega_{\mathrm{cr}}$, in close analogy with what is observed in MCs open clusters (e.g., Milone et al. 2018).

We first applied the procedure above to each synthetic CMD, by assuming that the eMSTO is due to age spread, and derived the FWHM of the age distribution. Results are represented with gray dots in Figure 11. As expected, the age spread increases with the cluster age, in close analogy with what was previously found by Niederhofer et al. (2015) in MCs clusters. The fact that the FWHM values derived for synthetic CMDs and for Galactic open clusters follow similar trends against the cluster age suggests that rotation is mainly responsible for the observed eMSTOs.

Finally, in Figures 12-13, we compare the CMDs of cluster members (left panels) with simulated CMDs (right panels). Synthetic CMDs are derived from the Geneva database 
(Georgy et al. 2014) and have metallicity, $Z=0.014$, and similar age, distance modulus, and reddening as those listed in Table 1. Unfortunately, rotating models are not available for stars less massive than $\sim 1.7 \mathcal{M}_{\odot}$. We note that, while in young clusters like NGC 2287 and NGC 2099 both fast rotators and slow-rotator stars are needed to reproduce the broad MS, the eMSTO of old clusters seems consistent with fast rotators alone. The poor quality of the fit could be due to the modeling of several second-order parameters that characterize the end of the core hydrogen burning phase, including the parameterization of the inclination angle, which strongly affects the stellar luminosity and effective temperature (see D'Antona et al. 2015 for details). Nevertheless, the comparison between data and simulations corroborates the conclusion that stellar rotation is mainly responsible for the observed eMSTOs and the broadened MSs.

\section{Summary and Discussion}

We have presented the first analysis of 12 open clusters in the Milky Way in the context of multiple stellar populations. Our results suggest that the multiple photometric sequences observed by Gaia in the CMDs of these nearby objects belong to the same phenomenon present in MCs clusters, and interpreted as due to stellar rotation and/or age spreads.

Since the early discoveries, the eMSTOs have been considered a common feature of the CMDs of LMC and SMC clusters younger than $\sim 2.5 \mathrm{Gyr}$ whereas the CMDs of Galactic open clusters were thought to be similar to simple isochrones. This picture has been challenged by the recent findings of eMSTOs in four Galactic open clusters younger than 1 Gyr, namely NGC 2099, NGC 2360, NGC 2818, and NGC 6705 (Bastian et al. 2018; Marino et al. 2018a).

We exploited the Gaia DR2 to analyze the CMDs of 12 Galactic open clusters younger than $\sim 1.5$ Gyr. We carefully separated cluster stars from field stars by using proper motions and parallaxes from Gaia DR2 and corrected the photometry of clusters members for differential reddening. We find that all the analyzed clusters show the eMSTO. In addition, all the clusters younger than $\sim 700 \mathrm{Myr}$ exhibit a broadened upper MS, whereas the bottom of the MS is narrow and well defined. The appearance of certain photometric features depending on age is similar to that observed in MCs clusters.

We statistically subtracted field stars with cluster-like proper motions and parallaxes from the CMD of candidate cluster stars, thus demonstrating that eMSTOs and broadened MSs are not due to residual contamination from field stars. We calculated for each cluster the synthetic photometry of a simple population of stars with the same age, metallicity, binary fraction, and observational errors. The comparison between the observations and the simulated CMDs reveals that the eMSTOs and the broadened MSs are due neither to observational uncertainties nor to unresolved binaries. These facts demonstrate that eMSTOs and broadened MSs are intrinsic features of the CMDs of the analyzed open clusters.

To investigate the physical mechanisms that are responsible for the eMSTO, we first compared the CMDs of cluster members with isochrones with different ages. The eMSTOs of the analyzed open clusters are consistent with stellar populations with different ages in close analogy with what has been observed in MC clusters with similar ages. The FWHM of the age spread ranges from about $70 \mathrm{Myr}$ in the $\sim 150 \mathrm{Myr}$ old cluster NGC 3114 to $\sim 260 \mathrm{Myr}$ in $\sim 1.1$ Gyr old NGC 5822 .
Interestingly, the derived age spread correlates with the cluster age, with old clusters having on average larger age spread than young clusters. A similar trend between the FWHM of the age distribution and the cluster age is present among MagellanicCloud clusters and is interpreted as an evidence that rotation is mainly responsible of the eMSTO. Indeed, in a simple stellar population, fast rotators appear younger than coeval nonrotating stars with the same age.

We compared the CMDs of cluster members with synthetic diagrams derived from Geneva models and find that the eMSTOs and the broadened MSs are consistent with coeval stellar populations with different rotation rates. These findings suggest that rotation is mainly responsible for the eMSTOs and the broadened MSs observed in Galactic clusters, and this corroborates direct spectroscopic evidence that stars with different rotation rates populate the eMSTOs of NGC 6705 and NGC 2818 (Bastian et al. 2018; Marino et al. 2018b) and that the blue and the red MS of NGC 6705 are populated by slow rotators and fast rotators, respectively (Marino et al. 2018b).

Our investigation of 12 Galactic open clusters demonstrates that the eMSTO and the broadened MS are not a peculiarity of MC star clusters but are common features of Galactic open clusters. Coeval stellar populations with different rotation rates are likely responsible for the eMSTO and the broadened MS of the analyzed clusters.

We thank the anonymous referee for several comments that improved the quality of this manuscript. This work has received funding from the European Research Council (ERC) under the European Union's Horizon 2020 research innovation programme (grant agreement ERC-StG 2016, No. 716082 "GALFOR"; PI: Milone), and the European Union' Horizon 2020 research and innovation programme under the Marie Skłodowska-Curie (grant Agreement No. 797100, beneficiary: Marino). A.P.M. acknowledges support from MIUR through the FARE project R164RM93XW "SEMPLICE"; A.F.M. has been supported by the Australian Research Council through Discovery Early Career Researcher Award DE160100851.

\section{ORCID iDs}

G. Cordoni (1) https://orcid.org/0000-0002-7690-7683

A. P. Milone (iD https://orcid.org/0000-0001-7506-930X

A. F. Marino (iD https://orcid.org/0000-0002-1276-5487

A. Dotter (10) https://orcid.org/0000-0002-4442-5700

E. P. Lagioia (1) https://orcid.org/0000-0003-1713-0082

\section{References}

Bastian, N., \& de Mink, S. E. 2009, MNRAS, 398, L11

Bastian, N., Kamann, S., Cabrera-Ziri, I., et al. 2018, MNRAS, 480, 3739

Casagrande, L., \& VandenBerg, D. A. 2018, MNRAS, 479, L102

Castelli, F., \& Kurucz, R. L. 2000, IAU, 210, 20

Claret, A. 2000, A\&A, 363, 1081

Conroy, C., \& Spergel, D. N. 2011, ApJ, 726, 36

Correnti, M., Goudfrooij, P., Bellini, A., Kalirai, J. S., \& Puzia, T. H. 2017, MNRAS, 467, 3628

D'Antona, F., Di Criscienzo, M., Decressin, T., et al. 2015, MNRAS, 453, 2637

D’Antona, F., Milone, A. P., Tailo, M., et al. 2017, NatAs, 1, 0186

D’Antona, F., Montalbán, J., Kupka, F., \& Heiter, U. 2002, ApJL, 564, L93

Dias, W. S., Alessi, B. S., Moitinho, A., \& Lépine, J. R. D. 2002, A\&A, 389,871

Dupree, A. K., Dotter, A., Johnson, C. I., et al. 2017, ApJL, 846, L1

Ekström, S., Georgy, C., Eggenberger, P., et al. 2012, A\&A, 537, A146 
Espinosa Lara, F., \& Rieutord, M. 2011, A\&A, 533, A43

Gaia Collaboration, Brown, A. G. A., Vallenari, A., et al. 2016, A\&A, 595, A2

Gaia Collaboration, Brown, A. G. A., Vallenari, A., et al. 2018, A\&A, 616, 1

Gallart, C., Zoccali, M., Bertelli, G., et al. 2003, AJ, 125, 742

Georgy, C., Granada, A., Ekström, S., et al. 2014, A\&A, 566, A21

Glatt, K., Grebel, E. K., Sabbi, E., et al. 2008, AJ, 136, 1703

Goudfrooij, P., Girardi, L., \& Correnti, M. 2017, ApJ, 846, 22

Goudfrooij, P., Girardi, L., Kozhurina-Platais, V., et al. 2014, ApJ, 797, 35

Goudfrooij, P., Puzia, T. H., Kozhurina-Platais, V., \& Chandar, R. 2011, ApJ, 737, 3

Keller, S. C., Mackey, A. D., \& Da Costa, G. S. 2011, ApJ, 731, 22

Li, C., de Grijs, R., Deng, L., \& Milone, A. P. 2017, ApJ, 844, 119

Mackey, A. D., \& Broby Nielsen, P. 2007, MNRAS, 379, 151

Mackey, A. D., Broby Nielsen, P., Ferguson, A. M. N., \& Richardson, J. C. 2008, ApJL, 681, L17

Marigo, P., Girardi, L., Bressan, A., et al. 2017, ApJ, 835, 77

Marino, A. F., Milone, A. P., Casagrande, L., et al. 2018a, ApJL, 863, L33
Marino, A. F., Milone, A. P., Yong, D., et al. 2014, MNRAS, 442, 3044

Marino, A. F., Przybilla, N., Milone, A. P., et al. 2018b, AJ, 156, 116

Milone, A. P., Bedin, L. R., Cassisi, S., et al. 2013, A\&A, 555, A143

Milone, A. P., Bedin, L. R., Piotto, G., et al. 2015, MNRAS, 450, 3750

Milone, A. P., Bedin, L. R., Piotto, G., \& Anderson, J. 2009, A\&A, 497, 755

Milone, A. P., Marino, A. F., Bedin, L. R., et al. 2016a, MNRAS, 455, 3009

Milone, A. P., Marino, A. F., D'Antona, F., et al. 2016b, MNRAS, 458, 4368

Milone, A. P., Marino, A. F., D'Antona, F., et al. 2017, MNRAS, 465, 4363

Milone, A. P., Marino, A. F., Di Criscienzo, M., et al. 2018, MNRAS, 477, 2640

Milone, A. P., Piotto, G., Bedin, L. R., et al. 2012, A\&A, 540, A16

Mowlavi, N., Eggenberger, P., Meynet, G., et al. 2012, A\&A, 541, A41

Niederhofer, F., Georgy, C., Bastian, N., \& Ekström, S. 2015, MNRAS, 453, 2070

Paunzen, E., Heiter, U., Netopil, M., \& Soubiran, C. 2010, A\&A, 517, A32

Sollima, A., Carballo-Bello, J. A., Beccari, G., et al. 2010, MNRAS, 401, 577

Yang, W., Bi, S., Meng, X., \& Liu, Z. 2013, ApJ, 776, 112 